\title{
Sequential rationalization of multivalued choice ${ }^{\text {光 }}$
}

\author{
María D. García-Sanz*, José Carlos R. Alcantud \\ Facultad de Economía y Empresa. Universidad de Salamanca. Campus Unamuno. \\ E37007 Salamanca, Spain.
}

\begin{abstract}
This paper contributes to the theory of rational choice under sequential criteria. Following the approach initiated by Manzini and Mariotti (2007) for single-valued choice functions, we characterize choice correspondences that are rational by two sequential criteria under a mild consistency axiom. Rationales ensuring the sequential rationalization are explicitly constructed and a uniquely determined, canonical solution is provided.
\end{abstract}

Keywords: choice correspondences, rational choice, sequential choice, compound correspondence, weak axiom of revealed preference.

JEL classification: D01, D71

\footnotetext{
This paper supersedes our manuscript "Rational choice by two sequential criteria", 2010 .

*Corresponding author. Tel: +34-923-294640. Fax: +34-923-294686

Email addresses: dgarcia@usal.es (María D. García-Sanz), jcr@usal.es (José Carlos R. Alcantud)

Preprint submitted to Mathematical Social Sciences

January 3, 2015
} 


\section{Introduction}

The literature on abstract choice theory abounds with analysis of the possible rationality of a choice function via satisfaction of consistency properties. The identification of rational choice as the maximizer of a unique preference has a long tradition in the literature (cf., Arrow (1959), Richter (1966, 1971), Wilson (1970), Sen (1971), Blair et al. (1976), Suzumura (1976, 1983), etc). But experimental economics has produced mounting evidence against the standard model of rational choice. Manzini and Mariotti (2007) provide a sample of references proving that cyclical choice occurs in simple decision problems on a regular basis. Among them, as early as Tversky (1969) gives a classical explanation of a cyclic pattern of choice: the alternatives have various relevant characteristics and we declare $a \succ b$ and $b \succ c$ although differences between consecutive alternatives are negligible in the foremost characteristic, but when we compare $a$ and $c$ their difference in such characteristic becomes noticeable and we declare $c \succ a$.

Our contribution is in continuation of a successful branch of the literature that studies decision makers (DMs) who choose by a sequential use of preferences that successively narrow the set of available alternatives. This model allows for cyclical choice and for violations of the usual menu independence properties. A focal reference is Manzini and Mariotti (2007), who analyse singled-valued choice functions that are sequentially rationalizable, i.e., the result of a sequential application of asymmetric binary relations or rationales. In addition they provide a complete analysis of the case of two and three relations. Apesteguía and Ballester (2013) investigate the restriction of sequential rationalizability to acyclic rationales, which is a more restrictive model: there are choice patterns that can be sequentially rationalized but cannot be sequentially rationalized by acyclic binary relations. Here we investigate another related model of choice, the differences being that $(i)$ we do not impose that the sequential application shrinks the set of available alternatives to exactly one final choice, $(i i)$ we do not impose that the rationales must be asymmetric, and (iii) we restrict our attention to the domain of choice correspondences -or multivalued choice functionsthat verify a mild version of the property of independence of irrelevant alternatives. Under such limitation, we characterize choice correspondences that are rationalized by the sequential application of two rational criteria. Our characterization is in terms of Expansion and a generalization for choice correspondences of the Weak WARP (Weak Axiom of Revealed Preference), thus replicating the characterization in Manzini and Mariotti (2007). When 
a solution exists, a uniquely determined, canonical solution is axiomatically and explicitly identified in terms of the economically relevant constructs, namely, the 'rationales'.

Multivalued choice functions play a distinctive role in this literature. Richter (1966) explicitly develops the revealed preference theory of consumer's behavior in terms of demand correspondences, rather than demand functions. Arrow (1959) and Sen (1971) extend WARP to choice correspondences. Mas-Colell et al. (1995), Section 1.C, explain that they can be used to capture the alternatives that the agent might choose. Thus for a set of alternatives $S$, we collect "those alternatives that we would actually see chosen if the decision maker were repeatedly to face the problem of choosing an alternative" from set $S$. Furthermore, the model of "choice from lists" in Rubinstein and Salant (2006) provides a new interpretation of the notion of choice correspondences: in the cases where the order in the list is not observable, choice correspondences permit to assign, to every set of alternatives, all the elements that are chosen for some ordering of that set.

The paper is organized as follows. In section 2 we set the notation and introduce our model. We recall some properties of rationality for choice correspondences and study their preservation by sequential application of two rational criteria. Section 3 contains our main result and a discussion on the uniqueness of the solution. In section 4 we conclude and comment on related literature.

\section{Definitions, properties of rationality and their preservation by sequential application}

Along this paper $X$ denotes a general set of alternatives and $\mathcal{P}(X)$ is the set of subsets of $X$. A binary relation on $X, R \subseteq X \times X$, is interpreted as a preference relation of an agent, that is $x R y($ or $(x, y) \in R$ ) if and only if the element $x \in X$ is considered at least as good as the element $y \in X$.

Definitions 1 and 2 below formalize the concepts of decisive choice correspondence and rational choice correspondence.

Definition 1. Let $\mathcal{D}$ be a nonempty domain of nonempty subsets of $X$, that is, $\varnothing \neq \mathcal{D} \subseteq \mathcal{P}(X)$ and $S \neq \varnothing$ for all $S \in \mathcal{D}$. A decisive choice correspondence on $\mathcal{D}$ is a map $\mathcal{C}: \mathcal{D} \rightarrow \mathcal{P}(X)$ such that $\mathcal{C}(S) \subseteq S$ and $\mathcal{C}(S) \neq \varnothing$ for all $S \in \mathcal{D}$. We also refer to (decisive) choice correspondences or multivalued choice functions as choice criteria. 
Unless otherwise stated, along this paper we are bound by two technical restrictions. Firstly, all choice correspondences are decisive thus choice correspondence holds for decisive choice correspondence. Secondly, $\mathcal{D}$ is the domain consisting of all finite and nonempty subsets of $X{ }^{1}$

Definition 2. A choice correspondence $\mathcal{C}$ on $\mathcal{D}$ is rational if there exists a binary relation $R$ on $X$ such that $\mathcal{C}(S)=\mathcal{C}_{R}(S)=\{x \in S: \forall y \in S,(x, y) \in$ $R\}$, for any set of alternatives $S \in \mathcal{D}$.

Definition 2 means that the choice is made by the optimization of a binary relation and we say that the binary relation $R$ rationalizes the choice correspondence $\mathcal{C}$. When $R$ is acyclic, then we say that the choice correspondence $\mathcal{C}$ is acyclic rational. As $\mathcal{D}$ contains all singletons and pairs of alternatives, any binary relation that rationalizes a decisive choice correspondence on $\mathcal{D}$ is reflexive and complete. Moreover, because $\mathcal{D}$ is the set of all finite and nonempty subsets of the set of alternatives, acyclic rational is equivalent to rational.

Rational correspondences are characterized by the following two properties (Blair et al. (1976)):

Definition 3. The choice correspondence $\mathcal{C}$ satisfies the Chernoff condition, also $\boldsymbol{C H}$, if for any $S, T \in \mathcal{D}$ such that $S \subseteq T$ we have $\mathcal{C}(T) \cap S \subseteq$ $\mathcal{C}(S){ }^{2}$

Definition 4. The choice correspondence $\mathcal{C}$ satisfies the Direct Condorcet property, also $\mathbf{C o n}^{+}$, if for any $S \in \mathcal{D}$ and $x \in S$ we have that $x \in \mathcal{C}(S)$ whenever $x \in \mathcal{C}(\{x, y\})$ for all $y \in S$.

Because we aim at identifying choice correspondences that arise from sequential application of rational choice correspondences, a crucial previous step is the analysis of the behavior of rationality under such sequential process formalized as follows:

Definition 5. Let $\mathcal{C}_{1}, \mathcal{C}_{2}$ be choice correspondences on $\mathcal{D}$. We define the composition of $\mathcal{C}_{1}$ and $\mathcal{C}_{2}$ as the map $\mathcal{C}_{2} \circ \mathcal{C}_{1}: \mathcal{D} \rightarrow \mathcal{P}(X)$ defined by:

$$
\left(C_{2} \circ C_{1}\right)(A)=C_{2}\left(C_{1}(A)\right) \text { for all } A \in \mathcal{D}
$$

\footnotetext{
${ }^{1}$ The results would not be affected if the domain includes all the infinite subsets as well, and even some of them are true for domains containing all pairs and all triples from $X$ only. We set this framework in order to avoid unnecessary technicalities.

${ }^{2}$ Equivalently: $\forall S, T \in \mathcal{D}, \mathcal{C}(S \cup T) \subseteq \mathcal{C}(S) \cup \mathcal{C}(T)$ whenever $S \cup T \in \mathcal{D}$ (Sertel and Bellem (1979)).
} 
The sequential application of rational choice functions is not necessarily rational since it produces violations of WARP. In fact it can explain cyclical patterns of choice, as already noted by Manzini and Mariotti (2007). We proceed to investigate when a choice correspondence can be obtained as the sequential application of two rational criteria, in the following terms:

Definition 6. A choice correspondence $\mathcal{C}$ on $\mathcal{D}$ is rational by two sequential criteria if there exist two rational choice correspondences $\mathcal{C}_{1}$ and $\mathcal{C}_{2}$ on $\mathcal{D}$ $\left(\mathcal{C}_{2}\right.$ being possibly indecisive) such that $\mathcal{C}=\mathcal{C}_{2} \circ \mathcal{C}_{1} \cdot{ }^{3}$

Manzini and Mariotti (2007) characterize choice functions $\mathcal{C}$ that are RSM (Rational Shortlist Methods), that is, for which there exist two asymmetric relations $R_{1}$ and $R_{2}$ such that $\mathcal{C}(S)=\mathcal{C}_{R_{2}}\left(\mathcal{C}_{R_{1}}(S)\right)$. Two axioms are jointly necessary and sufficient for RSM, namely, Expansion and Weak WARP. We state them in their general form for choice correspondences:

Definition 7. A choice correspondence $\mathcal{C}$ on $\mathcal{D}$ satisfies the Expansion property ${ }^{4}$, also $\boldsymbol{E}$, if for any collection of subsets $\left\{M_{i}\right\}_{i \in I}$ in $\mathcal{D}, x \in$ $\mathcal{C}\left(M_{i}\right)$ for all $i \in I$ entails $x \in \mathcal{C}\left(\cup_{i \in I} M_{i}\right)$.

Definition 8. A choice correspondence $\mathcal{C}$ satisfies the Weak WARP property, also WWARP, if for any $x, y \in X$ and $S, T \in \mathcal{D}$ such that $\{x, y\} \subseteq$ $S \subseteq T, \mathcal{C}(\{x, y\})=\{x\}$ and $x \in \mathcal{C}(T) \Rightarrow y \notin \mathcal{C}(S)$.

It is known that $\mathbf{E}$ and $\mathbf{C H}$ are independent properties. Manzini and Mariotti (2007) prove the independence of WWARP and E. Example 1 in the Appendix shows that properties WWARP and $\mathbf{E}$ do not imply $\mathbf{C H}$.

As expected, Manzini and Mariotti's characterization does not translate faithfully to choice correspondences. To be precise, Proposition 1 below proves that when we compound two rational choice correspondences we obtain a choice correspondence that satisfies $\mathbf{E}$ and WWARP. However Example 2 in the Appendix proves that these axioms do not suffice to characterize choice correspondences rational by two sequential criteria.

Proposition 1. If $\mathcal{C}_{1}$ and $\mathcal{C}_{2}$ are rational choice correspondences on $\mathcal{D}$, then the compound choice correspondence $\mathcal{C}=\mathcal{C}_{2} \circ \mathcal{C}_{1}$ satisfies $\boldsymbol{E}$ and $\boldsymbol{W} \boldsymbol{W A R P}$.

\footnotetext{
${ }^{3}$ Observe that $\mathcal{C}_{2}$ must be nonempty-valued on $\left\{\mathcal{C}_{1}(S): S \in \mathcal{D}\right\}$.

${ }^{4}$ This is Sen's Property $\gamma$.
} 
Proof. Let $R_{1}$ and $R_{2}$ be two binary relations such that $\mathcal{C}_{1}=\mathcal{C}_{R_{1}}$ and $\mathcal{C}_{2}=\mathcal{C}_{R_{2}}$. We proceed to check that $\mathcal{C}$ satisfies property $\mathbf{E}$.

Let $\left\{S_{i}\right\}_{i \in I}$ be a collection of sets in $\mathcal{D}$ such that $x \in \mathcal{C}\left(S_{i}\right)$ for all $i \in I$. We must prove that $x \in \mathcal{C}\left(\bigcup_{i \in I} S_{i}\right)$. Since $x \in \mathcal{C}_{R_{2}}\left(\mathcal{C}_{R_{1}}\left(S_{i}\right)\right)$ for all $i \in I$, $x \in \mathcal{C}_{R_{1}}\left(S_{i}\right)$ for all $i \in I$. This, together with the fact that $\mathcal{C}_{1}$ satisfies $\mathrm{Con}^{+}$, leads to

$$
x R_{1} y \text { for all } y \in S_{i} \forall i \in I \Rightarrow x R_{1} y \forall y \in \bigcup_{i \in I} S_{i} \Rightarrow x \in \mathcal{C}_{R_{1}}\left(\bigcup_{i \in I} S_{i}\right)
$$

If $x \notin \mathcal{C}_{R_{2}}\left(\mathcal{C}_{R_{1}}\left(\bigcup_{i \in I} S_{i}\right)\right)$, then there exists $z \in \mathcal{C}_{R_{1}}\left(\bigcup_{i \in I} S_{i}\right)$ such that $\neg\left(x R_{2} z\right)\left(\mathcal{C}_{2}\right.$ satisfies $\left.\mathbf{C o n}^{+}\right)$.

In addition, $z \in \mathcal{C}_{R_{1}}\left(\bigcup_{i \in I} S_{i}\right)$ implies that $z \in \mathcal{C}_{R_{1}}\left(S_{i}\right)$ for some $i \in I$ (because $\mathcal{C}_{1}$ satisfies $\mathbf{C H}$ ). From $x \in \mathcal{C}_{R_{2}}\left(\mathcal{C}_{R_{1}}\left(S_{i}\right)\right)$ for all $i \in I$ we obtain $x R_{2} z$, a contradiction that leads to $x \in \mathcal{C}\left(\bigcup_{i \in I} S_{i}\right)$.

We now prove that $\mathcal{C}$ satisfies WWARP. Let us select $\{x, y\} \subseteq S \subseteq T$ such that $\{x\}=\left(\mathcal{C}_{R_{2}} \circ \mathcal{C}_{R_{1}}\right)(\{x, y\})$ and $x \in\left(\mathcal{C}_{R_{2}} \circ \mathcal{C}_{R_{1}}\right)(T)$. We show that $y \notin \mathcal{C}(S)$ by contradiction. In case $y \in \mathcal{C}(S)$ we have

$(y, s) \in R_{1}$ for all $s \in S$ and $\left(y, s^{\prime}\right) \in R_{2}$ for all $s^{\prime} \in S$ such that $s^{\prime} R_{1} s$ for all $s \in S$

From $x \in\left(\mathcal{C}_{R_{2}} \circ \mathcal{C}_{R_{1}}\right)(T)$ we obtain $(x, t) \in R_{1}$ for all $t \in T$. In particular:

i) $(y, x) \in R_{1}$ because $x \in S$, and $(x, y) \in R_{1}$ because $y \in T$, which implies that $\mathcal{C}_{R_{1}}(\{x, y\})=\{x, y\}$.

ii) $(y, x) \in R_{2}$ because $x R_{1} t$ for all $t \in T$ and $S \subseteq T$, which means $y \in \mathcal{C}_{R_{2}}(\{x, y\})$.

Thus $y \in \mathcal{C}_{R_{2}}(\{x, y\})=\mathcal{C}_{R_{2}}\left(\mathcal{C}_{R_{1}}(\{x, y\})\right)=\mathcal{C}(\{x, y\})$, against the assumption. This concludes the proof.

\section{Rationalizability by two sequential criteria}

In view of the discussion in Section 2, the following questions arise: what properties permit to solve Manzini and Mariotti's problem in the case of multivalued choices? When a solution exists, can we give a uniquely determined or canonical solution? We address these issues in separate subsections. 


\subsection{Main result}

We proceed to prove that on a mildly restricted class of choice correspondences, the testable conditions $\mathbf{E}$ and WWARP are sufficient to ensure an exact realization by two sequential criteria. In other words, we obtain a characterization of rationalizability by two sequential criteria for multivalued choices verifying a weak property that we call 'choosing without dominated elements'. This property boils down to the elimination of dominated elements in the presence of dominating elements, in the following terms:

Definition 9. Let $\mathcal{C}$ be a choice correspondence on $\mathcal{D}$. An alternative $x \in X$ is dominated by another alternative $y \in X$ if $x$ is never chosen when $y$ is available. That is, for any $S \subseteq X$ such that $\{x, y\} \subseteq S$ we have $x \notin \mathcal{C}(S)$.

One cannot find a finite cycle of domination, due to decisiveness. Observe that $\mathcal{C}(\{x, y, z, \ldots, t\})=\varnothing$ when $x$ is dominated by $y, y$ is dominated by $z, \ldots, t$ is dominated by $x$ (for any $x, y, z, \ldots, t \in S$ ), which is absurd.

Definition 10. Let $\mathcal{C}$ be a choice correspondence on $\mathcal{D}$. It satisfies the property of choosing without dominated elements (CWDE) if given two elements $x, y$ such that $x$ is dominated by $y$ and $x, y \in S \in \mathcal{D}$, then $\mathcal{C}(S)=\mathcal{C}(S \backslash\{x\})$.

Remark 1. It is simple to check that if the elements $x_{1}, \ldots, x_{k} \in S \in \mathcal{D}$ are dominated (by respective $y_{1}, \ldots, y_{k} \in S$ ) then $\mathcal{C}(S)=\mathcal{C}\left(S \backslash\left\{x_{1}, \ldots, x_{k}\right\}\right)$. The reason is that the absence of cycles of domination permits to arrange these elements so that each $x_{i}$ is dominated by either $x_{j}$ with $j>i$, or by $z_{i} \in S \backslash\left\{x_{1}, \ldots, x_{k}\right\}$. Therefore $\mathcal{C}(S)=\mathcal{C}\left(S \backslash\left\{x_{1}\right\}\right)=\mathcal{C}\left(S \backslash\left\{x_{1}, x_{2}\right\}\right)=$ $\ldots=\mathcal{C}\left(S \backslash\left\{x_{1}, \ldots, x_{k}\right\}\right)$ follows immediately.

Some choice procedures are inconsistent with maximization of a preference due to violations of the property of independence of irrelevant alternatives (IIA). CWDE is a consistency axiom about the final choice behavior that brings the ethos of the IIA axiom back into the picture in the analysis of sequential rationalizability: it requests that if an alternative is chosen from a set, it remains chosen when dominated alternatives are discarded from the set, and conversely. In line with Manzini and Mariotti's original interpretation, under CWDE the DM considers everything but chooses as if alternatives dominated by others had been eliminated. 
Before proceeding with our characterization it is convenient to define the following complete binary relations on a set $X$ (associated with a choice correspondence $\mathcal{C}$ on $\mathcal{D}$ that satisfies $\mathbf{C o n}^{+}$):

$$
\begin{gathered}
x R_{1} y \Leftrightarrow \exists S^{\prime} \in \mathcal{D}: x, y \in S^{\prime} \text { and } x \in \mathcal{C}\left(S^{\prime}\right) \\
x R_{2} y \Leftrightarrow x \in \mathcal{C}(\{x, y\})
\end{gathered}
$$

It is clear that $\mathcal{C} \subseteq \mathcal{C}_{R_{1}}$, and property Con $^{+}$ensures $\mathcal{C}_{R_{2}} \subseteq \mathcal{C}$. In short, if $\mathcal{C}$ is a choice correspondence satisfying $\mathbf{C o n}^{+}$then $\mathcal{C}_{R_{1}}$ is a rational choice correspondence containing $\mathcal{C}$ and $\mathcal{C}_{R_{2}}$ is a rational choice correspondence contained in $\mathcal{C}$.

Aizerman and Aleskerov (1995), Section 5.5, deal with these relations in the context of approximation of choice functions. They prove that for any rational choice correspondence $\tilde{\mathcal{C}}$ such that $\tilde{\mathcal{C}} \subseteq \mathcal{C}$ it must be the case that $\tilde{\mathcal{C}} \subseteq \mathcal{C}_{R_{2}} \subseteq \mathcal{C}$. Similarly, for any rational choice correspondence $\overline{\mathcal{C}}$ such that $\mathcal{C} \subseteq \overline{\mathcal{C}}$ it must be the case that $\mathcal{C} \subseteq \mathcal{C}_{R_{1}} \subseteq \overline{\mathcal{C}}$. In conclusion, $\mathcal{C}_{R_{1}}$ is the smallest rational correspondence containing $\mathcal{C}$ and $\mathcal{C}_{R_{2}}$ is the greatest rational correspondence contained in $\mathcal{C}$.

We are now ready to prove our main result:

Theorem 1. Let $\mathcal{C}$ be a choice correspondence that verifies $\boldsymbol{C W D E}$. Then $\mathcal{C}$ is rational by two sequential criteria if and only if $\mathcal{C}$ verifies $\boldsymbol{E}$ and WWARP.

Proof. Proposition 1 proves necessity without the recourse to CWDE. To prove sufficiency let $\mathcal{C}$ be a choice correspondence satisfying $\mathbf{C W D E}, \mathbf{E}$ and WWARP. In order to show that there exist $R_{1}$ and $R_{2}$ such that $\mathcal{C}=\mathcal{C}_{R_{2}} \circ \mathcal{C}_{R_{1}}$, we consider the binary relations defined by equations (1) and (2) above.

We first check $\mathcal{C} \subseteq \mathcal{C}_{R_{2}} \circ \mathcal{C}_{R_{1}}$. If $x \in \mathcal{C}(S)$ then $x \in \mathcal{C}_{R_{1}}(S)$. Now let us suppose that $x \notin \mathcal{C}_{R_{2}}\left(\mathcal{C}_{R_{1}}(S)\right)$. In this case there exists $y \in \mathcal{C}_{R_{1}}(S)$ such that $\{y\}=\mathcal{C}(\{x, y\})$ by definition of $\mathcal{C}_{R_{2}}$. From $y \in \mathcal{C}_{R_{1}}(S)$ we obtain that for all $s \in S$ there exists $S_{y s} \in \mathcal{D}$ such that $y, s \in S_{y s}$ and $y \in \mathcal{C}\left(S_{y s}\right)$.

As $\mathcal{C}$ satisfies property $\mathbf{E}$ we conclude $y \in \mathcal{C}\left(\cup_{s \in S} S_{y s}\right)$.

Then we have $\{x, y\} \subseteq S \subseteq \cup_{s \in S} S_{y s}$ with $\{y\}=\mathcal{C}(\{x, y\})$ and $y \in$ $\mathcal{C}\left(\cup_{s \in S} S_{y s}\right)$. 
Applying WWARP we conclude that $x \notin \mathcal{C}(S)$, against the hypothesis. Therefore $x \in \mathcal{C}_{R_{2}}\left(\mathcal{C}_{R_{1}}(S)\right)$.

To complete the demonstration it remains to check that $\mathcal{C}_{R_{2}} \circ \mathcal{C}_{R_{1}} \subseteq \mathcal{C}$. In fact it suffices to check that $\left(\mathcal{C}_{R_{2}} \circ \mathcal{C}_{R_{1}}\right)(S) \subseteq \mathcal{C}\left(\mathcal{C}_{R_{1}}(S)\right)$ because $\mathcal{C}\left(\mathcal{C}_{R_{1}}(S)\right)=$ $\mathcal{C}(S)$ due to the $\mathbf{C W D E}$ assumption on $\mathcal{C}$ (see Remark 1). Let us select $x \in \mathcal{C}_{R_{2}}\left(\mathcal{C}_{R_{1}}(S)\right)$. If $x \notin \mathcal{C}\left(\mathcal{C}_{R_{1}}(S)\right)$ then there is $y \in \mathcal{C}_{R_{1}}(S)$ such that $\mathcal{C}(\{x, y\})=\{y\}$, because $\mathcal{C}$ satisfies $\mathbf{E}$. This implies that $x \notin \mathcal{C}_{R_{2}}\left(\mathcal{C}_{R_{1}}(S)\right)$ which is impossible.

\subsection{A uniquely determined canonical representation}

In Theorem 1 we prove that two explicitly defined rationales convey the rationalization of a choice correspondence satisfying CWDE, E and WWARP by two sequential criteria, which is an economically relevant fact in the tradition of revealed preference theory. We cannot assure uniqueness of the rationalizing relations. However this seems to be a minor concern because our solution in terms of the $R_{1}$ and $R_{2}$ rationales delivers a uniquely determined canonical representation, because as we have noticed above, $\mathcal{C}_{R_{2}}(S) \subseteq \mathcal{C}(S) \subseteq \mathcal{C}_{R_{1}}(S)$ for any $S \in \mathcal{D}$ and $\mathcal{C}_{R_{1}}$ is the smallest rational choice correspondence that contains $\mathcal{C}$, and $\mathcal{C}_{R_{2}}$ is the greatest rational choice correspondence contained in $\mathcal{C}$.

In conclusion, under the proper circumstances we have both explicit and axiomatic descriptions of a uniquely determined solution to the problem of rationalizability by two sequential criteria.

\section{Concluding remarks and related literature}

Our research contributes to a growing literature that applies the revealed preference approach to the study of individual choices that arise from criticism to the classical postulate of maximization of one well-behaved preference. For any given model of choice, the traditional interpretation of the rationalizability of the observables is that we identify choice functions/correspondences that could be explained as if the agent behaves according to such model. This is our emphasis, rather than the debate whether or not agents actually behave in that way.

In the most permissive approach, Kalai et al. (2002) observe that choice functions are always rationalized by a collection of rationales (RMR or rationalization by multiple rationales), such that for every choice problem in the domain, the unique choice is maximal in the problem for some rationale 
in the collection. And therefore they focus on those RMR that employ the minimal number of orderings.

More precisely, our research relates to a recent body of work that studies models where the final choice comes after elimination of items, especially when rationales prune the set of alternatives. Manzini and Mariotti (2007) prove a characterization of the Rational Shortlist Method although they leave unanswered what can be inferred about the underlying rationales from observed behavior. Dutta and Horan (2014) subsequently study to what extent and how observed behavior pins down the underlying rationales in the RSM model. Thus they are concerned by issues that we do not address here either: an exact characterization of all pairs of rationales that represent a given choice function, and a uniqueness result that is original in this analysis (to wit, a characterization of the transformations of any representation that induce the same choice behavior). Au and Kawai (2011) study transitive-RSMs, a model more restrictive than RSMs that requires transitivity for both rationales. It is characterized by an axiom that rules out choice functions that reveal a cyclic preference relation, although the transitive-RSM model allows for cyclic behavior too. In a different vein, Apesteguía and Ballester (2013) show that choice with a status quo bias (Masatlioglu and Ok (2005)) is a refinement of sequential rationalizability, which can be described in terms of their sequential procedure guided by routes. Houy (2007) analyses whether the order of the criteria affects the final choice or not in the model by sequential rationalizability with unrestricted rationales. He proves that in the case of choice functions, the orderindependence of sequentially rational choices is necessary and sufficient to explain rational choices. Relatedly, lexicographic heuristics have also raised the interest of e.g., Houy and Tadenuma (2009), who study two distinct procedures of choice by lexicographic application of multiple criteria, and Manzini and Mariotti (2012), who develop a model of boundedly rational choice that generalizes ideas in Tversky (1969) and explore its connection with sequential rationalizability. Among earlier contributions in this line, Aizerman and Malishevski (1986) study when a two stage process can be reduced to a one stage case having the same solution, and Aleskerov and Cinar (2008) study the representability by a one-stage scalar extremization model of a particular two-stage model in which the first stage selects alternatives that are dominated by no more than $q$ alternatives and the second one is a scalar extremization model. In this trend the assumption is made that the agent is aware of all available alternatives and (acts as if he) 
consciously eliminates some of them, a case of limited consideration.

Various models involving the use of consideration sets in decision theory have been proposed to capture other possible sources of limited consideration, e.g., ( $i$ ) unawareness or bounded understanding of what is available as in Masatlioglu et al. (2012), (ii) excessive abundance of alternatives as in Lleras et al. (2011), or even (iii) psychological restrictions as in Cherepanov et al. (2013). A consideration set is the subset of all available options to which the DM pays attention. Masatlioglu et al. (2012) provide a choice theoretical foundation for maximizing a complete and transitive relation under limited attention. One behavioral postulate of choice called WARP(LA) characterizes their model of "choice with limited attention". The consideration sets in their model must be inferred from the DM's final choice. Relatedly, Masatlioglu and Nakayima (2013) are concerned with an environment where it is not realistic to assume that the DM knows the entire feasible set of alternatives. Therefore they deal with a consideration set that evolves during the course of search because the DM must explore it. Lleras et al. (2011) explicitly define limited consideration sets to capture systematic failures to consider available options due to the paradox of choice. ${ }^{5}$ A single behavioral principle called LC-WARP characterizes their model of "choice with limited consideration" (CLC) in the case of choice functions. Cherepanov et al. (2013) study DMs who have preferences over alternatives and a set of rationales (modeled as binary relations). The DM chooses the alternative she prefers among the feasible options that are optimal according to at least one of her rationales, called the permissible options. Put differently, she makes her choice from each subset among its permissible alternatives only.

We consider choice correspondences that verify the following mild consistency axiom: choice is unaffected by removing an alternative that would never be chosen when some others are available. As is common in this literature, we assume that choices from every possible problem are known. Then we extend the characterization of sequential rationality by two criteria given by Manzini and Mariotti (2007) for choice functions. We prove that choice correspondences in our class are rational by two sequential criteria if and only if they satisfy the properties of Expansion and a generalization of their $W W A R P$ for choice correspondences. ${ }^{6}$

\footnotetext{
${ }^{5} \mathrm{~A}$ particular specification of their model for single-valued choice is a strict subclass of the RSM, therefore of our model.

${ }^{6}$ Tyson (2013) provides a different Generalized WWARP for choice correspondences
} 
Of course the use of not necessarily single-valued choice correspondences in models with multiple criteria is not original to our work. We have commented upon the model of "choice from lists" in Rubinstein and Salant (2006) and its interplay with choice correspondences. ${ }^{7}$ Houy (2007) shows that the extension of his aforementioned characterization to choice correspondences is no longer true although the necessity part of the statement remains valid. Demuynck (2011) studies the computational complexity of various choice models that use multiple rationales to explain observed choice behavior in the form of choice correspondences, which includes the case of Apesteguía and Ballester (2010). Lleras et al. (2011) separately consider the analysis of their model of CLC with multivalued choices in an Appendix. Now they need two axioms to characterize CLC. ${ }^{8}$ Dutta and Horan (2014) conclude by hinting that their main results on identification of rationales can be extended to choice correspondences, which would complement our work in the same sense that their proven results for choice functions complement Manzini and Mariotti (2007). However their uniqueness argument crucially hinges on the property that choices are univalued. Tyson (2013) considers "two-stage shortlisting procedures in which the menu of alternatives is first pruned by a process or criterion and then a binary relation is maximized", and he proves a meta-characterization in a formal setting that is completely devoid of background assumptions like single-valued choice or domain restrictions. In particular, his analysis accepts multivalued choice outputs from any unrestricted collection of menus. The assumption of an exhaustive dataset has also been criticized by de de Clippel and Rozen (2012) both from an empirical and experimental standpoint. These authors attempt to provide a better understanding of how to test bounded rationality theories when datasets are limited. They show that Samuelson's revealed preference approach can generalize to theories of bounded rationality in the analysis of choice functions.

There is also an emerging literature that applies the revealed preference approach to the study of collective decisions. Similarly to our approach,

in his Condition 3.20.

${ }^{7}$ In a related line, Yildiz (2012) characterizes list rational choice functions, that is, choice functions for which there exist a list and a binary relation in such a way that the DM makes his choice by successive comparisons of the alternatives according to the binary relation and following the list.

${ }^{8}$ To this purpose, the natural modification of their original axiom LC-WARP extends the concept of revealed indifference. An earlier treatment of revealed indifference in choice theory can be found in Alcantud (2002). 
the main aim is to identify the testable restrictions of theories of collective decision-making when individual preferences are not observable. A fundamental work in the abstract context -i.e., without the restrictions of earlier contributions to for example, household behavior or the general equilibrium model- is Sprumont (2010). A recent reference with a nice review of the literature dealing with noncooperative game-theoretic solution concepts is Bossert and Sprumont (2013), who prove that every choice function is backwards-induction rationalizable. The extension of this result to choice correspondences has been independently proven by Rehbeck (2014) and Xiong (2014).

\section{Acknowledgements}

We are very grateful to the Editor of the journal, Jean-François Laslier, and an anonymous referee for helpful suggestions. We also acknowledge valuable comments from Paola Manzini, Marco Mariotti and Kemal Yildiz. This work is financially supported by the Spanish Ministerio de Economía y Competitividad under Project ECON2012-31933.

\section{Bibliography}

M.A. Aizerman, A.V. Malishevski, 1986. Conditions for Universal Reducibility of a Twostage extremization problem to a one-stage problem. J. Math. Anal. Appl. 119, 361388.

M.A. Aizerman, F. Aleskerov, 1995. Theory of Choice. North-Holland, Amsterdam.

J.C.R. Alcantud, 2002. Revealed indifference and models of choice behavior. J. Math. Psychol. 46(4), 418-430.

F. Aleskerov, Y. Cinar, 2008. ' $q$-Pareto-scalar' two stage extremization model and its reducibility to one-stage model. Theory and Decision 65, 325-338.

J. Apesteguía, M.A. Ballester, 2010. The computational complexity of rationalizing behavior. J. Math. Econ. 46, 356-363.

J. Apesteguía, M.A. Ballester, 2013. Choice by sequential procedures. Games Econ. Behav. 77, 90-99.

K.J. Arrow, 1959. Rational choice functions and orderings. Economica 26, 121-127.

P.H. Au, K. Kawai. Sequentially rationalizable choice with transitive rationales. Games Econ. Behav. 73 (2), 608-614

D.H. Blair, G. Bordes, J.S. Kelly, K. Suzumura, 1976. Impossibility Theorems without Collective Rationality. J. Econ. Theory 13, 361-379.

W. Bossert, Y. Sprumont, 2013. Every choice function is backwards-induction rationalizable. Econometrica 81, 2521-2534.

V. Cherepanov, T. Feddersen, A. Sandroni, 2013. Rationalization. Theor. Econ. 8, 775800 . 
G. de Clippel, K. Rozen, 2012. Bounded Rationality and Limited Databases: Testable Implications, Identifiability, and Out-of-Sample Prediction. Cowles Foundation Discussion Paper No. 1853.

T. Demuynck, 2011. The computational complexity of rationalizing boundedly rational choice behavior. J. Math. Econ. 47, 425-433.

R. Dutta and S. Horan, Inferring Rationales from Choice: Identification for Rational Shortlist Methods, 2014. Unpublished.

N. Houy, 2007. Rationality and order-dependent sequential rationality. Theory Dec. 62(2), 119-134.

N. Houy, K. Tadenuma, 2009. Lexicographic compositions of multiple criteria for decision making. J. Econ. Theory 144, 1770-1782.

G. Kalai, A. Rubinstein, R. Spiegler, 2002. Rationalizing choice functions by multiple rationales. Econometrica 70(6), 2481-2488.

J.S. Lleras, Y. Masatlioglu, D. Nakajima, and E. Ozbay, 2011. When More is Less: Choice with Limited Consideration. Unpublished.

P. Manzini, M. Mariotti, 2007. Sequentially rationalizable choice. Amer. Econ. Rev. 97, 1824-1839.

P. Manzini, M. Mariotti, 2012. Choice by lexicographic semiorders. Theor. Econ. 7, 1-23.

Y. Masatlioglu, E. A. Ok, 2005. Rational choice with status quo bias. J. Econ. Theory $121(1), 1-29$.

Y. Masatlioglu, D. Nakajima, E.Y. Ozbay, 2012. Revealed Attention. Amer. Econ. Rev. 102(5), 2183-2205.

Y. Masatlioglu, D. Nakajima, 2013. Choice by iterative search. Theor. Econ. 8, 701-728.

A. Mas-Colell, M. D. Whinston, J. R. Green, 1995. Microeconomic Theory. Oxford University Press.

J. Rehbeck, 2014. Every choice correspondence is backwards-induction rationalizable. Working paper.

M.K. Richter, 1966. Revealed Preference Theory. Econometrica 34(3), 635-645.

M.K. Richter, 1971. Rational choice, in: J.S. Chipman, L. Hurwicz, M.K. Richter, H.F. Sonnenchein (Eds.), Preferences, Utility, and Demand. Harcourt Brace Jovanovich, New York.

A. Rubinstein, Y. Salant, 2006. A model of choice from lists. Theor. Econ. 1, 3-17.

A. Sen, 1971. Choice functions and revealed preference. Rev. Econ. Stud. 38, 307-312.

M.R. Sertel, A.V.D. Bellem, 1979. Synopses in the theory of choice. Econometrica 47(6), 1367-1389.

Y. Sprumont, 2010. On the testable implications of collective choice theories. J. Econ. Theory 93, 205-232.

K. Suzumura, 1976. Rational Choice and Revealed Preference. Rev. Econ. Stud. 43, 149-158.

K. Suzumura, 1983. Rational Choice, Collective Decisions, and Social Welfare. Cambridge University Press. Cambridge.

A. Tversky, 1969. Intransitivity of preferences. Psycohol. Rev. 76, 31-48.

C.J. Tyson, 2013. Behavioral implications of shortlisting procedures. Soc. Choice and Welfare 41, 941-963.

R.B. Wilson, 1970. The finer structure of revealed preference. J. Econ. Theory 2, 348-353.

S. Xiong, 2014. Every choice correspondence is backwards-induction rationalizable. Work- 
K. $\begin{aligned} & \text { ing paper. } \\ & \text { Yildiz, 2012. List rationalizable choice. Available at SSRN: }\end{aligned}$ http://ssrn.com/abstract $=2147231$. 


\section{Appendix}

Example 1. There exist choice correspondences satisfying $\boldsymbol{E}$ (and therefore $\mathbf{C o n}^{+}$) and WWARP, but not $\boldsymbol{C H}$. The choice correspondence $\mathcal{C}$ on $\mathcal{P}^{*}(X)$ when $X=\{x, y, z\}$ given by Table 1 proves this assertion: it fails to verify $\boldsymbol{C H}$ because $\{y, z\} \subseteq\{x, y, z\}$ but $\mathcal{C}(\{x, y, z\}) \cap\{y, z\}=\{y\} \nsubseteq$ $\mathcal{C}(\{y, z\})=\{z\}$.

Table 1. $\mathcal{C}$ satisfies $\boldsymbol{E}$ and $\boldsymbol{W} \boldsymbol{W A R P}$, but not $\boldsymbol{C H}$

\begin{tabular}{ccccc}
\hline$S$ & $\{x, y\}$ & $\{x, z\}$ & $\{y, z\}$ & $\{x, y, z\}$ \\
$\mathcal{C}(S)$ & $\{x, y\}$ & $\{x\}$ & $\{z\}$ & $\{x, y\}$ \\
\hline
\end{tabular}

Example 2. There exist choice correspodences that satisfy $\boldsymbol{E}$ and $\boldsymbol{W} \boldsymbol{W A R P}$ and are not rational by two sequential criteria. The choice correspondence $\mathcal{C}$ on $\mathcal{P}^{*}(X)$ when $X=\{x, y, z, t, s\}$ given by Table 2 proves this assertion.

Table 2. $\mathcal{C}$ satisfies $\boldsymbol{E}$ and $\boldsymbol{W} \boldsymbol{W A R P}$ and is not rational by two sequential criteria

\begin{tabular}{ccccccc}
\hline$S$ & $\{x, y\}$ & $\{x, z\}$ & $\{x, t\}$ & $\{x, s\}$ & $\{y, z\}$ & $\{y, t\}$ \\
$\mathcal{C}(S)$ & $\{y\}$ & $\{x, z\}$ & $\{x, t\}$ & $\{x, s\}$ & $\{z\}$ & $\{y\}$ \\
\hline$S$ & $\{y, s\}$ & $\{z, t\}$ & $\{z, s\}$ & $\{t, s\}$ & $\{x, y, z\}$ & $\{x, y, t\}$ \\
$\mathcal{C}(S)$ & $\{s\}$ & $\{z, t\}$ & $\{z\}$ & $\{t\}$ & $\{z\}$ & $\{y\}$ \\
\hline$S$ & $\{x, y, s\}$ & $\{x, z, t\}$ & $\{x, z, s\}$ & $\{x, t, s\}$ & $\{y, z, t\}$ & $\{y, z, s\}$ \\
$\mathcal{C}(S)$ & $\{s\}$ & $\{x, z, t\}$ & $\{x, z\}$ & $\{x, t\}$ & $\{z\}$ & $\{z\}$ \\
\hline$S$ & $\{y, t, s\}$ & $\{z, t, s\}$ & $\{x, y, z, t\}$ & $\{x, y, z, s\}$ & $\{x, y, t, s\}$ & $\{x, z, t, s\}$ \\
$\mathcal{C}(S)$ & $\{t\}$ & $\{z, t\}$ & $\{z, t\}$ & $\{x, z\}$ & $\{x, t\}$ & $\{x, z, t\}$ \\
\hline$S$ & $\{y, z, t, s\}$ & $\{x, y, z, t, s\}$ & & & & \\
$\mathcal{C}(S)$ & $\{z, t\}$ & $\{x, z, t\}$ & & & & \\
\hline
\end{tabular}

$A$ direct inspection shows that $\mathcal{C}$ satisfies properties $\boldsymbol{E}$ and WWARP. To prove that it is not rational by two sequential criteria, assume by way of contradiction that $\mathcal{C}_{1}$ and $\mathcal{C}_{2}$ are rational choice correspondences such that $\mathcal{C}=\mathcal{C}_{2} \circ \mathcal{C}_{1}$

It must be the case that $x, z, t \in \mathcal{C}_{1}(\{x, y, z, t, s\})$ and applying that $\mathcal{C}_{1}$ satisfies $\boldsymbol{C H}, x, z, t \in \mathcal{C}_{1}(\{x, y, z, t\})$. Now two possibilities arise: 
1. $\mathcal{C}_{1}(\{x, y, z, t\})=\{x, y, z, t\}$.

In this case, $\mathcal{C}=\mathcal{C}_{2} \circ \mathcal{C}_{1}$ implies that $\mathcal{C}_{2}(\{x, y, z, t\})=\{z, t\}$. Because $\mathcal{C}_{1}$ and $\mathcal{C}_{2}$ satisfy $\boldsymbol{C H}$ one has $\mathcal{C}_{1}(\{y, t\})=\{y, t\}$ and $t \in \mathcal{C}_{2}(\{y, t\})$, which leads to $t \in\left(\mathcal{C}_{2} \circ \mathcal{C}_{1}\right)(\{y, t\})$ thus $\left(\mathcal{C}_{2} \circ \mathcal{C}_{1}\right)(\{y, t\}) \neq \mathcal{C}(\{y, t\})$.

2. $\mathcal{C}_{1}(\{x, y, z, t\})=\{x, z, t\}$.

From $\mathcal{C}=\mathcal{C}_{2} \circ \mathcal{C}_{1}$ we obtain $\mathcal{C}_{2}(\{x, z, t\})=\{z, t\}$. Because $\mathcal{C}_{1}$ satisfies $\boldsymbol{C H}$ one has $\mathcal{C}_{1}(\{x, z, t\})=\{x, z, t\}$ thus $\left(\mathcal{C}_{2} \circ \mathcal{C}_{1}\right)(\{x, z, t\})=\{z, t\} \neq$ $\mathcal{C}(\{x, z, t\})$. 